\title{
Flexural properties of electrospun polymethyl methacrylate microfiber-reinforced BisGMA for dental post prefabrication
}

\author{
Nina Djustiana ${ }^{1,2^{*}}$, Yanwar Faza ${ }^{1,2}$, Arief Cahyanto ${ }^{2,3}$ \\ 'Department of Dental Materials Science and Technology, Faculty of Dentistry Universitas \\ Padjadjaran, Indonesia \\ ${ }^{2}$ Oral Biomaterials Study Centre, Faculty of Dentistry Universitas Padjadjaran, Indonesia \\ ${ }^{3}$ Department of Restorative Dentistry, Faculty of Dentistry University of Malaya, Malaysia
}

\begin{abstract}
Introduction: A dental post is a restoration to preserve the remaining tooth structure thus can be functioned normally. Many researchers suggested a fiber dental post due to its biomechanical properties that are similar to dentin structure. This study aims to analyse the flexural properties of electrospun polymethyl methacrylate microfiber-reinforced BisGMA for dental post prefabrication. Methods: The sample used was following the ADA guideline, as well as for the number of samples. The sample size was $25 \times 2 \times 2 \mathrm{~mm}$, which is close to the average dental post size. PMMA microfibers were prepared by dissolving heat cure PMMA powder with $99 \%$ acetone, then electrospinning with a rotary collector. Acquired PMMA microfibers were immersed into the resin matrix containing BisGMA, camphorquinone, and 2-dimethylaminoethyl methacrylate (DMAEMA) as a monomer, initiator, and co-initiator, respectively, to prepare the dental posts. Results: PMMA microfibers structure and surface fracture of dental posts were confirmed by Scanning Electron Microscopy (SEM). PMMA microfibers show unaligned fiber morphology with an approximate diameter size of 1-5 $\mu \mathrm{m}$. A universal testing machine was used to measure the dental post's flexural properties (flexural strength and flexural modulus). Dental posts with PMMA fibers showed higher flexural strength $(83.5 \pm 10.7 \mathrm{MPa})$ compared to the dental post without PMMA fibers (61.7 $\pm 3.03 \mathrm{MPa}$ ) with a $\mathrm{p}$-value $<0.05$. On the other hand, PMMA fibers' addition did not significantly increase the dental post's flexural modulus. Conclusion: The PMMA microfibers can intimately adhere to the BisGMA mixture as the resin matrix. Therefore, the PMMA microfiber significantly improves the flexural strength of the BisGMA for dental post prefabrication.
\end{abstract}

Keywords: Dental post, flexural properties, polymethyl methacrylate, BisGMA

p-ISSN: 1979-0201; e-ISSN: 2549-6212; Available from: http://jurnal.unpad.ac.id/pjd/article/view/15894

DOI: 10.24198/pid.vol33no1.15894

Submission: Feb 26, 2018; Accepted: Nov 30, 2021; Published online: Nov 30, 2021

*Corresponding author: Nina Djustiana, Department of Dental Materials Science and Technology, Faculty of Dentistry Universitas Padjadjaran, Indonesia. Jalan Sekeloa Selatan I, Bandung, West Java, 40132, Indonesia. Phone: +62 812-2036868; e-mail: nina.djustiana@fkg.unpad.ac.id 


\section{INTRODUCTION}

A dental post is a treatment to rebuild a tooth structure that lost a massive portion of the tooth after endodontic treatment. It can function as a solid restorative unit together with the dental crown. $^{1-3}$ Nowadays, fiber dental posts were introduced to the market, and it is known as less stiff and possesses acceptable stress distribution in remaining dentin structure, thus hindering fracture incident. ${ }^{4,5}$ Fiber dental post is one type of dental post that contains a high volume percentage of continuous fibers in a methacrylatebased resin, for instance, BisGMA. This kind of dental post is more aesthetic than metal post and ceramic post. Therefore, it is more applicable to be used for anterior crown restoration. ${ }^{2}$ However, despite the advantages of fiber as a dental post material, its ability to enhance the mechanical properties of the dental post is still controversial. Some studies show positive results while others do not. ${ }^{1}$

PMMA is a type of material that can be used as a reinforcing material for methacrylatebased resins because both are known to be chemically bonded. ${ }^{6}$ PMMA fibers have the following characteristics, such as biocompatible, transparent, colorless, easy to handle, good processing ability, low cost, and high surface area to volume ratio. $7,8,910$ However, the utilization of PMMA fiber as a reinforcing material for methacrylate-based resins has not been widely found

It is well known that there is a relationship between the enhancing mechanical properties and characteristics of fiber beneath the resin matrix. The characteristics are type, size, integrity, density, distribution, configuration, and the bond between the fibers and the matrix. ${ }^{11,12}$ Studies show that characteristics play a role in determining the flexural strengths of the fiber dental posts. The flexural strength represents the fracture resistance of the specimens toward flexural stresses. It is determined by the highest load the specimen can retain and related to the specimen configuration. ${ }^{13,14}$ The selection of materials that can produce interfacial bonds needs to be considered because the discontinuity in the interface and the existence of the void within the resin may ruin the mechanical properties of fiber dental posts. ${ }^{11,15}$ In this study, the author proposes to synthesis the PMMA fiber in micron size using the electrospinning method. This method has several advantages such as simple procedure, easy to control the size, and alignment of the fibers. ${ }^{16,17}$ Upon synthesis of the PMMA microfiber, it is used to be a reinforcing material of BISGMA for the dental post prefabrication then analyze its flexural properties via flexural strength and modulus value and interface evaluation.

\section{METHODS}

Polymethyl methacrylate (PMMA) heat cured (Triplex Hot) was purchased from a local dental store in Bandung, Indonesia. Bisphenol-A-Glycidyl Methacrylate (BisGMA), champhorquinone (CQ), 2-(dimethylamino)ethyl methacrylate (DMAEMA) were purchased from Sigma-Aldrich, Singapore. PMMA $0.6 \mathrm{~g}$ were dissolved into $20 \mathrm{ml}$ acetone 99\% (concentration of pmma in the solvent was $38.5 \%)$. The solution was stirred for 30 minutes by a magnetic stirrer until PMMA dissolved. The solution was then electrospun with a rotary collector and aluminum foil sheet on it. A flow rate of $0.8 \mathrm{~mL} / \mathrm{h}$ set electrospun, $13.8 \mathrm{kV}$ applied voltage, $12 \mathrm{~cm}$ distance between needle and collector, and $45 \%$ chamber moisture.

\section{Fabrication of Electrospun PMMA microfibers reinforced BisGMA as dental post prefabrication} The BisGMA (98.5 wt\%) was mechanically mixed with initiator CQ $(0.5 \% \mathrm{wt})$ and co-initiator DMAEMA ( $1 \mathrm{wt} \%)$ at $70{ }^{\circ} \mathrm{C}$ for 1 hour. Microfibers were cut into pieces with $25 \mathrm{~mm} \times 2 \mathrm{~mm}$ size for a three-point bending test. BisGMA mixture was poured into Teflon mold of $25 \mathrm{~mm} \times 2 \mathrm{~mm} \times$ $2 \mathrm{~mm}$ until a half mold was filled, then laid up PMMA microfibers on top of the mixture. BisGMA and PMMA microfibers were mixed mechanically by spatula, then continued by adding the BisGMA mixture on top until throughout mold was fulfilled. Put mylar strip over the mold, press with a glass slide, then light-cured using LED (SmarLite Focus, Dentsply Sirona, Charlotte, USA) for 20 seconds. Withdraw the glass slide and mylar strip, continue light-cured for another 20 seconds. The specimens were then removed from the mold then stored at $37{ }^{\circ} \mathrm{C}$ for 24 hours. Dental posts prefabrication containing BisGMA mixture without PMMA 
microfiber were also prepared with the same steps as the control. The number of test specimens and control specimens is 6 samples each.

\section{Morphology of PMMA microfibers}

The PMMA microfibers were characterized by Scanning Electron Microscopy (SEM), with specifications as the follows: Hitachi S-4700, Tokyo, Japan, to identify the morphologies and particle size of the PMMA microfibers. The fracture surface was also observed by SEM characterization to see interfacial bonding between PMMA microfibers with resin BisGMA.

Functional group evaluation by Fourier Transform Infrared Spectroscopy (FTIR)

The PMMA microfibers specimen were ground to obtain fine powder for FTIR analysis. A ratio of 1:200 (specimen and $\mathrm{KBr}$ ) were mixed well and compacted in stainless steel die. A spectrometer (Spectrum 100, PerkinElmer Inc., Shelton, USA) uses a spectral resolution of $4 \mathrm{~cm}^{-1}$ and a range of wavenumber from $400-4000 \mathrm{~cm}^{-1}$.

\section{Flexural properties evaluation using Universal Testing Machine}

The specimens and controls with $\mathrm{n}=6$ were tested in three-point bending by a universal testing machine (Lloyd Instrument, Fareham, Hampshire, UK) for flexural strength test (according to ISO 10477 standard) with a span of $20 \mathrm{~mm}$ and crosshead speed $1 \mathrm{~mm} / \mathrm{min}$.

The following formula was used to calculate the flexural strength (1) and modulus (2):

$\mathrm{F}_{\mathrm{s}}=3 \mathrm{FI} / 2 \mathrm{bh}^{2}$.

(1)

$F_{y}=\mid 3 F_{1} / 4 \mathrm{fbh}^{3}$.

(2)

$\mathrm{F}$ is the applied load at the highest point $(\mathrm{N})$; $\mathrm{I}$ is the span length $(20 \mathrm{~mm}) ; \mathrm{b}$ is the width of specimens; $h$ is the thickness of the specimens; $F_{1}$ is the load at the convenient point in the straightline portion of the trace; $f$ is the deflection of the specimens at load $\mathrm{F}_{1}$.

\section{Data analysis}

Statistical analysis was carried out using an unpaired t-test by KaleidaGraph 4.1 (Synergy Software, PA, USA) to ensure the statistical significance noted experimentally.

\section{RESULTS}

\section{Morphology view of PMMA microfibers}

The SEM image in Figure 1 exhibits the characteristic morphology of PMMA fibers. It was shown unaligned fiber morphology with a relatively homogeneous size. The diameter size of PMMA fibers was ranging from 1- 5 microns.

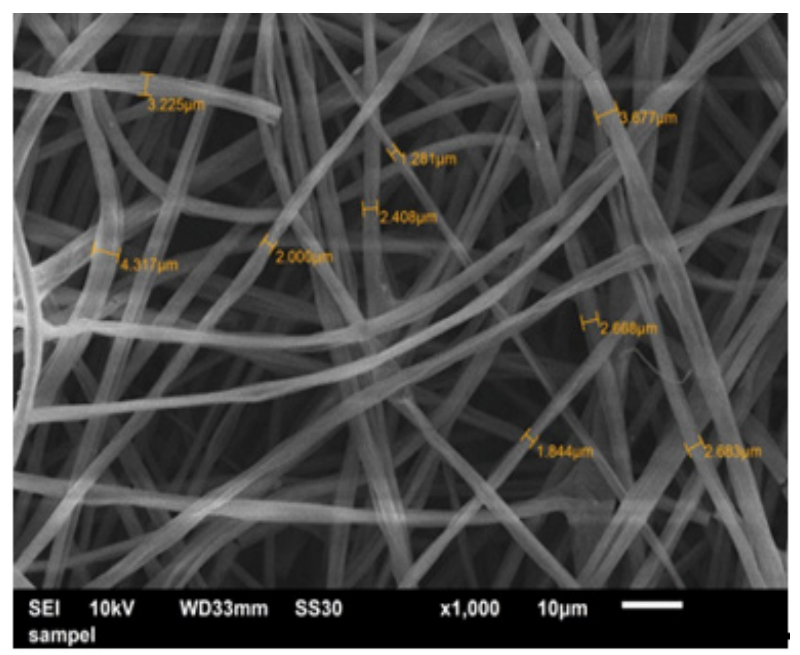

Figure 1. SEM image of the electrospun PMMA microfiber

Functional group detection of PMMA microfibers Figure 2 shows the spectra of the PMMA microfibers by infrared spectroscopy. The peak at $2950 \mathrm{~cm}^{-1}$ corresponds to the stretching of the methacryloyl group $\left(\mathrm{CH}_{3}\right.$ and $\left.\mathrm{CH}_{2}\right)$. The typical PMMA is shown by the presence of carbonyl group in $1722 \mathrm{~cm}^{-1}$ with a strong peak. The peaks correspond to the $\mathrm{C}-\mathrm{O}$ and $\mathrm{CH}_{2}$ vibrates at 1142 and $749 \mathrm{~cm}^{-1}$. At the same time, the peak of $985 \mathrm{~cm}^{-1}$ attributed to the $\mathrm{C}-\mathrm{C}$ group.

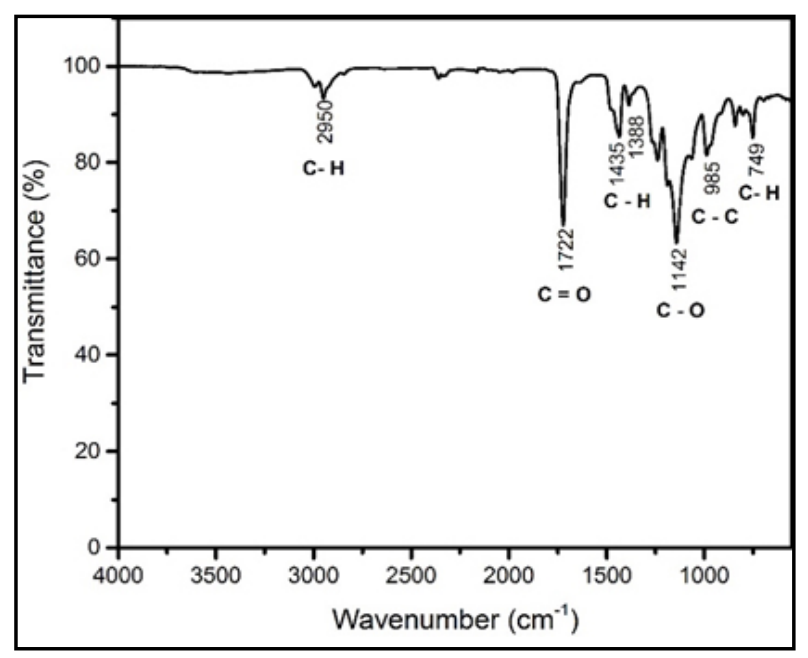

Figure 2. FTIR spectra of PMMA microfibers 
Flexural properties of the dental posts with and without PMMA microfibers

Table 1 shows the data normality test of flexural strength and flexural modulus values in the dental post fabrication group with and without PMMA. The results of the normality test show that both groups have data values that are normally distributed ( $p$ $>0.05$ ) in both tests using Saphiro-Wilk Test.

Table 1. Test the normality of the two data groups on the flexural strength and flexural modulus value using the Shapiro-Wilk test

\begin{tabular}{ccc}
\hline Groups & Flexural strength $(\mathrm{MPa})^{*}$ & Flexural Modulus $(\mathrm{GPa})^{*}$ \\
\hline Without PMMA $(\mathrm{n}=6)$ & 0.271 & 0.206 \\
With PMMA $(\mathrm{n}=6)$ & 0.432 & 0.380 \\
\hline
\end{tabular}

Notes: ${ }^{*} p>0.05$

Table 2 summarizes the flexural strength and flexural modulus of the dental post prefabrication with and without PMMA microfibers. Dental posts with PMMA microfibers showed higher flexural strength compared with the dental post without
PMMA microfibers. The PMMA microfibers increase the flexural strength of dental posts by $35.3 \%$. However, the addition of PMMA microfibers did not show increasing in flexural modulus in the dental post.

Table 2. The flexural strength and modulus value of the sample without and with PMMA. The statistical analysis was done by unpaired t-test

\begin{tabular}{ccc}
\hline Groups & Flexural strength $(\mathrm{MPa})^{*}$ & Flexural Modulus $(\mathrm{GPa})^{*}$ \\
\hline Without PMMA $(\mathrm{n}=10)$ & $61.7 \pm 3.03$ & $3.1 \pm 1.0$ \\
With PMMA $(\mathrm{n}=10)$ & $83.5 \pm 10.7$ & $5.0 \pm 2.9$ \\
\hline
\end{tabular}

Notes: * $p>0.05$

\section{Interface evaluation of dental post with and without PMMA microfibers}

Figure 3 shows the dental post's fracture surface without PMMA (a) and with PMMA microfibers (b). Dental post prefabrication without PMMA microfiber presents a smooth surface and few rough spots after fracturing. On the contrary, the fracture surface for a dental post prefabrication with PMMA microfibers looks very rough than another group.

Although the shape in the image looks like the fiber is not very visible in the image below, this shows that the formation of chemical bonds between PMMA and BisGMA fibers.
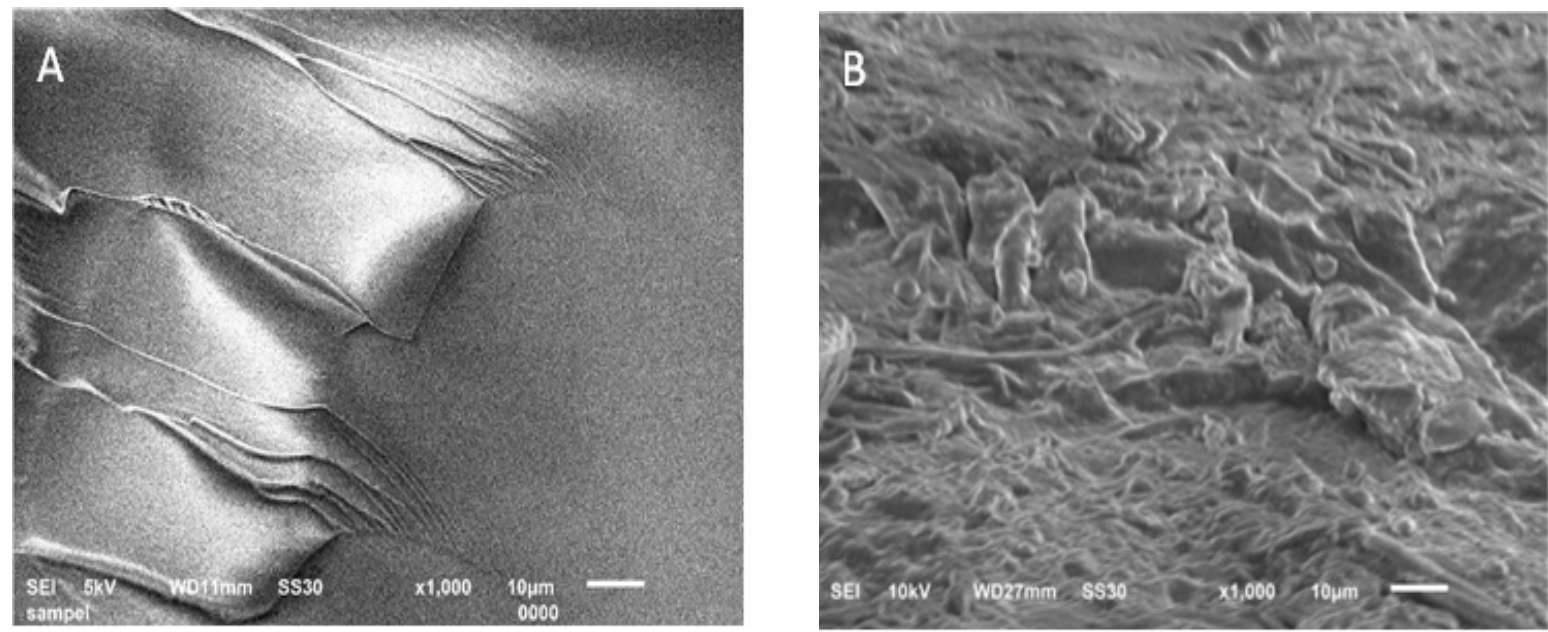

Figure 3. SEM images of the dental post's fracture surface; (A) without PMMA microfibers and (B) with PMMA microfibers 


\section{DISCUSSION}

Electrospinning is commonly used to produce nano-and micron-sized fibers. This method not only controls the size but also the alignment. Ura et al. tailored 3.6 $\mu \mathrm{m}$ PMMA microfibers using a $30 \%$ PMMA concentration. ${ }^{10}$ This is similar to the concentration used in this study which is $38 \%$ then maybe that is why the size was also similar which is $1-5 \mu \mathrm{m}$ (Fig 1 ). The support theory remarks that PMMA concentration affects the formation of fiber, for instance, lower concentration $(\leq 12 \%)$ tends to obtain nano-sized fiber, and higher concentration $(\geq 30 \%)$ tends to obtain micron size fiber. ${ }^{10}$

Subsequently, the surface of acquired PMMA fibers is smooth with unaligned orientation. The unaligned fiber occurs due to fluid instability during the electrospinning process. It is known as splaying or branching, which leads the jet to undergo splitting into multiple jets. This would change the shape of the jet and charge per unit area as compensation for the jet elongation and evaporation. As a result, the unstable jet will eject the smaller one from the surface of the primary jet. ${ }^{18}$

BisGMA reinforced with PMMA microfibers improved the flexural strength but not the modulus elasticity (Table 2). The elevation of flexural strength is related to the effect of the fibers. The fibers provide a high ratio of the specific area to resist flexural stress, impeding the formation of cracks. On the contrary, the flexural modulus values for both groups are similar. There are no statistical differences between those groups. It is due to flexural strength mainly relying on the fibers' strength; however, the flexural modulus mainly relies on the matrix. ${ }^{19}$ Previous studies stated that the ultrahigh specific nanofiber area is risky to decrease the flexural properties. It will create inferior impregnation, leading to air entrapment formation.

Therefore, increasing the mass fraction of fibers to further improvement will be difficult. Hinder the defect is the critical point to elevate the flexural properties. Flexural strength tends to decrease with defects increasing. ${ }^{13}$ The interfacial adhesion between the microfibers and the matrix was identified in Fig. 3. The SEM image shows there are no microfibers that were maintaining their configuration in the resin matrix. Although not entirely true, the authors suspect that the PMMA fibers were partly dissolved into the resin matrix. This phenomenon is similar to the study of Cheng et al. where the PMMA-containing fiber exhibited a borderless image between the fiber and the resin matrix. ${ }^{14}$ This is due to the favorable interaction between BisGMA and PMMA by methacryloyl groups $\left(\mathrm{CH}_{3}\right.$ and $\left.\mathrm{CH}_{2}\right)$ on each of their chains. The FTIR result (Fig. 2) shows the methacryloyl groups belonging to PMMA microfibers are identified at $2950 \mathrm{~cm}^{-1}$.

These groups play the role of forming a semiIPN structure with resin matrix after curing. ${ }^{14,20}$. Based on the things above, the authors suspect the formation of a good interfacial bond between the PMMA microfiber and the resin matrix, thereby increasing the value of the flexural strength of the dental post prefabrication. Since the microfibers are strongly bonded to the resin matrix, the frictional force arises following stress transfer across the matrix, increasing the flexural strength. $13,14,20$

This study shows that the type of fiber and fiber-matrix interfacial bond, PMMA, can increase the flexural strength of dental posts prefabrication, but the effect of size and configuration of PMMA fiber cannot be clearly identified because further research is needed. The results of the study show that PMMA polymer has the potential as a material used as a reinforcement material for dental posts prefabrication.

\section{CONCLUSIONS}

The PMMA microfibers can intimately adhere to the BisGMA mixture thus significantly improving the flexural strength of the dental post prefabrication. However, PMMA microfibers do not increase the flexural modulus where this property is more influenced by the resin matrix used.

\section{ACKNOWLEDGMENT}

The authors are grateful for the financial support for this study from Universitas Padjadjaran, Indonesia.

\section{REFERENCES}

1. Shah S, Shilpa-Jain DP, Velmurugan N, 
Sooriaprakas C, Krithikadatta J. Performance of fibre reinforced composite as a postendodontic restoration on different endodontic cavity designs- an in-vitro study. J Mech Behav Biomed Mater 2020;104:103650. DOI: 10.1016/j.jmbbm.2020.103650.

2. Machado J, Almeida P, Fernandes S, Marques A, Vaz M. Currently used systems of dental posts for endodontic treatment. Procedia Struct Integr. 2017. p. 5, 27-33.

3. Djustiana N, Faza $Y$, Karlina E, Hasratiningsih Z, Munir MM, and Khairurrijal K. Flexural Strength Evaluation of Dental Post Prototype Contain ZAS-PMMA Composite Fiber with Electrospinning Methods. Key Eng Mater. 2020; 829: 93-9. DOI:10.4028/www. scientific. net/KEM.829.93.

4. Almaroof AG, Thyab SA, Ali AH. Bond strength of a new Kevlar fiber-reinforced composite post with semi-interpenetrating polymer network (IPN) matrix. J Clin Exp Dent 2019; 11(8): e695-700. DOI: 10.4317/jced.55703.

5. de Almeida LN, Mendes GAM, Favarao IN, Kasuya AVB, Barges MG, Menezes MdS, et al. Influence of preheating and post-curing on a novel fiber-reinforced composite post material. Braz Oral Res 2018; 32: 1-13. DOI:10.1590/1807-3107bor-2018.vol32.0097

6. Hatta M, ShinyaA, Gomi H, Vallittu PK, Säilynoja E, Lassila LVJ. Effect of Interpenetrating Polymer Network (IPN) Thermoplastic Resin on Flexural Strength of Fibre-Reinforced Composite and the Penetration of Bonding Resin into Semi-IPN FRC Post. Polymers (Basel). 2021. p. 13.

7. Philip P, Tomlal Jose E, Chacko JK, Philip KC Thomas PC. Preparation And Characterisation Of Surface Roughened PMMA Electrospun Nanofibers From PEO - PMMA Polymer Blend Nanofibers. Polym Test. 2019. p. 74, 257-65.

8. Dong H, Strawhecker KE, Snyder JF, Orlicki J A, Reiner RS, Rudie AW. Cellulose Nanocrystals As A Reinforcing Material For Electrospun Poly(Methyl Methacrylate) Fibers: Formation, Properties And Nanomechanical Characterization. Carbohydr Polym. 2012. p. 87, 2488-95.

9. Djustiana N, Faza Y, Sudiyasari N, Firdaus AT, Usri K, Cahyanto A. Performance of Electrospun PMMA-Silica Nanofiber as Reinforced Material in Dental Composite Restoration. J Int Dent Med Res, 2020; 13: 975-8.

10. Ura DP, Karbowniczek JE, Szewczyk PK, Metwally S, Kopyściański M, Stachewicz, U. Cell Integration with Electrospun PMMA Nanofibers, Microfibers, Ribbons, and Films: A Microscopy Study. Bioeng. (Basel, Switzerland), 2019. p. 6, 41.

11. Novais VR, Rodrigues RB, Simamoto Júnior, PC, Lourenço CS, Soares CJ. Correlation between the mechanical properties and structural characteristics of different fiber posts systems. Braz Dent J 2016; 27: 46-51. DOI: 10.1590/0103-6440201600377.

12. Wandscher VF, Bergoli CD, de Oliveira AF, Kaizeret OB, Borges ALS, Limberguer IDF, et al. Fatigue surviving, fracture resistance, shear stress and finite element analysis of glass fiber posts with different diameters. J Mech Behav Biomed Mater 2015; 43: 69-77. DOI: 10.1016/j.jmbbm.2014.11.016.

13. Vidotti HA, Manso AP, Leung V, do Valle AL, Ko F, Carvalho RM. Flexural properties of experimental nanofiber reinforced composite areaffectedby resincompositionandnanofiber/ resin ratio. Dent Mater 2015;31:1132-41. DOI: 10.1016/j.dental.2015.06.018.

14. Cheng L, Zhou X, Zhong H, Deng X, Cai Q, Yang $X$. NaF-loaded core-shell PAN-PMMA nanofibers as reinforcements for Bis-GMA/TEGDMA restorative resins. Mater. Sci. Eng. C. Mater Biol Appl 2014; 34: 262-9. DOI: 10.1016/j. msec.2013.09.020.

15. Zicari F, Coutinho E, Scotti R, Van Meerbeek B, Naert I. Mechanical properties and micro-morphology of fiber posts. Dent Mat 2013;29:e45-52. DOI: 10.1016/j. dental.2012.11.001.

16. Xue J, Wu T, Dai Y, Xia Y. Electrospinning and Electrospun Nanofibers: Methods, Materials, and Applications. Chem Rev 2019; 119: 5298415. DOI: $10.1021 /$ acs.chemrev.8b00593.

17. Wang $X$, Ding $B$, Sun $G$, Wang $M$, Yu J. Electrospinning/netting: A strategy for the fabrication of three-dimensional polymer nano-fiber/ nets. Prog Mater Sci 2013; 58(8): 1173-243. DOI: $10.1016 / j . p m a t s c i .2013 .05 .001$

18. Garg K, Bowlin GL. Electrospinning jets and nanofibrous structures. Biomicrofluidics 2011;5:13403. DOI: $10.1063 / 1.3567097$ 
19. Özyürek T, Topkara C, Koçak I, Yılmaz K, Gündoğar M, Uslu G. Fracture Strength Of Endodontically Treated Teeth Restored With Different Fiber Post And Core Systems. Odontology, 2020. p. 108, 588-95.
20. Canché-Escamilla G, Duarte-Aranda S, Toledano M. Synthesis And Characterization Of Hybrid Silica/PMMA Nanoparticles And Their Use As Filler In Dental Composites. Mater. Sci. Eng. C. Mater Biol Appl 2014. p. 42, 161-7. 